\title{
Comparisons of CODE and CNES/CLS GPS satellite bias products and applications in Sentinel-3 satellite precise orbit determination
}

\author{
Bingbing Duan ${ }^{1}\left[\right.$. $\cdot$ Urs Hugentobler $^{1}$
}

Received: 30 December 2020 / Accepted: 10 July 2021 / Published online: 16 July 2021

(c) The Author(s) 2021

\begin{abstract}
To resolve undifferenced GNSS phase ambiguities, dedicated satellite products are needed, such as satellite orbits, clock offsets and biases. The International GNSS Service CNES/CLS analysis center provides satellite (HMW) Hatch-MelbourneWübbena bias and dedicated satellite clock products (including satellite phase bias), while the CODE analysis center provides satellite OSB (observable-specific-bias) and integer clock products. The CNES/CLS GPS satellite HMW bias products are determined by the Hatch-Melbourne-Wübbena (HMW) linear combination and aggregate both code (C1W, C2W) and phase (L1W, L2W) biases. By forming the HMW linear combination of CODE OSB corrections on the same signals, we compare CODE satellite HMW biases to those from CNES/CLS. The fractional part of GPS satellite HMW biases from both analysis centers are very close to each other, with a mean Root-Mean-Square (RMS) of differences of 0.01 wide-lane cycles. A direct comparison of satellite narrow-lane biases is not easily possible since satellite narrow-lane biases are correlated with satellite orbit and clock products, as well as with integer wide-lane ambiguities. Moreover, CNES/CLS provides no satellite narrow-lane biases but incorporates them into satellite clock offsets. Therefore, we compute differences of GPS satellite orbits, clock offsets, integer wide-lane ambiguities and narrow-lane biases (only for CODE products) between CODE and CNES/CLS products. The total difference of these terms for each satellite represents the difference of the narrow-lane bias by subtracting certain integer narrow-lane cycles. We call this total difference "narrow-lane" bias difference. We find that $3 \%$ of the narrow-lane biases from these two analysis centers during the experimental time period have differences larger than 0.05 narrow-lane cycles. In fact, this is mainly caused by one Block IIA satellite since satellite clock offsets of the IIA satellite cannot be well determined during eclipsing seasons. To show the application of both types of GPS products, we apply them for Sentinel-3 satellite orbit determination. The wide-lane fixing rates using both products are more than $98 \%$, while the narrow-lane fixing rates are more than 95\%. Ambiguity-fixed Sentinel-3 satellite orbits show clear improvement over float solutions. RMS of 6-h orbit overlaps improves by about a factor of two. Also, we observe similar improvements by comparing our Sentinel-3 orbit solutions to the external combined products. Standard deviation value of Satellite Laser Ranging residuals is reduced by more than $10 \%$ for Sentinel-3A and more than $15 \%$ for Sentinel-3B satellite by fixing ambiguities to integer values.
\end{abstract}

Keywords Bias comparison $\cdot$ Sentinel-3A/B $\cdot$ Undifferenced ambiguity resolution $\cdot$ CNES/CLS $\cdot$ CODE

Bingbing Duan

bingbing.duan@tum.de

Urs Hugentobler

urs.hugentobler@tum.de

1 Institute for Astronomical and Physical Geodesy, Technical University of Munich, Arcisstr 21, 80333 Munich, Germany

\section{Introduction}

The IGS (International GNSS Service) has been providing precise GPS satellite orbit and clock products for more than 20 years (Johnston et al. 2017). This service enables the PPP (precise point positioning) technique that allows a single receiver to achieve centimeter to millimeter level positioning accuracy for daily static solutions (Malys and Jensen 1990; Zumberge et al. 1997; Kouba and Héroux 2001). After a good understanding of biases from satellites and receivers, the PPP-AR (ambiguity resolution) technique 
is then possible. To serve PPP-AR applications, a number of IGS analysis centers have developed their own satellite bias products, for instance, CNES/CLS (Center National d'Etudes Spatiales/ Collecte Localisation Satellites), CODE (Center for Orbit Determination in Europe), EMR (Natural Resources Canada), ESA (European Space Agency), GFZ (GeoForschungsZentrum), JPL (Jet Propulsion Laboratory), TUG (Graz University of Technology), TUM (Technical University of Munich) and WUHN (Wuhan University) (Duan et al. 2021; Ge et al. 2005; Geng et al. 2012; Li et al. 2018; Loyer et al. 2012; Schaer et al. 2018; Uhlemann et al. 2015). To combine bias products from individual analysis centers, a new IGS working group (PPP-AR) was created at the IGS workshop 2018 in Wuhan, China. As shown by Banville et al. (2020), a preliminary combination of satellite bias and clock products from six analysis centers over one week is confirmed to be successfully achieved.

The PPP-AR technique is carried out by fixing widelane and narrow-lane ambiguities sequentially. CNES/CLS provides GPS satellite HMW bias products based on C1W, $\mathrm{C} 2 \mathrm{~W}, \mathrm{~L} 1 \mathrm{~W}$, and L2W signals in the Hatch-MelbourneWübbena (HMW) linear combination and provides dedicated satellite clock products, including satellite narrowlane biases. Since 2018 CNES/CLS satellite products have been extended to Galileo satellites as well (Katsigianni et al. 2019). From December 2019, the CODE analysis center started to provide GPS/Galileo satellite orbit, integer clock and OSB products (Bock et al. 2009; Schaer et al. 2018; Villiger et al. 2019). Different than providing satellite HMW and narrow-lane biases, CODE provides bias products for individual signals. This contribution compares these two types of GPS satellite bias products and applies both of them for Sentinel-3 satellite orbit determination.

The Sentinel satellite missions are next-generation earth observation missions operated by the European Commission (EC) and the European Space Agency (ESA) as part of the Copernicus program. The goal is to support the joint ESA/ EC initiative GMES (Global Monitoring for Environment and Security) (Aschbacher 2017). For example, to ensure accurate GMES services, orbit determination of Sentinel-3 satellites has a targeted uncertainty of less than $2 \mathrm{~cm}$ in the radial direction. The Sentinel-3 spacecraft, which are the focus of this research, hosts a POD (precise orbit determination) package comprising GPS and DORIS (Doppler Orbitography and Radiopositioning Integrated by Satellite) receivers and antennas, as well as a laser retroreflector (Jalabert and Mercier 2018; Štěpánek et al. 2020). The official precise Sentinel-3 satellite orbits are made available by the Copernicus Precise Orbit Determination (CPOD) Quality Working Group (QWG) based on onboard GPS measurements (Fernandez et al. 2016, 2014; Montenbruck et al. 2018; Peter et al. 2017). Kinematic (Jäggi et al. 2016) and reduced-dynamic (Bertiger et al. 1994, 2010; Bock et al.
2014; Flohrer et al. 2011; Montenbruck et al. 2005; Van Den IJssel et al. 2015) methods are optionally adopted by individual analysis centers within the CPOD QWG.

As part of CPOD QWG, we routinely generate Sentinel satellite orbit products based on the reduced-dynamic method. At the beginning, we used CODE final GPS satellite orbit and 5-s clock products. Sentinel data sampling was $10 \mathrm{~s}$ and we provided float-ambiguity orbit solutions. Mid of 2018, the half-cycle issue of carrier phase observations was corrected in the Sentinel-3 RINEX (Receiver INdependent EXchange format) file, and we started to provide ambiguity-fixed Sentinel-3 satellite orbits using CNES/CLS satellite products. To avoid long-time interval satellite clock interpolation, we reduced measurement sampling to $30 \mathrm{~s}$, the clock product sampling provided by CNES/CLS. From the end of 2019, CODE published GPS satellite phase bias and 5-s sampling clock products. We can compare the performances of both types of products for Sentinel-3 satellite orbit determination. Also, we have the possibility to increase the Sentinel-3 data sampling again to $10 \mathrm{~s}$. Therefore, the main goal of this contribution is as follows. First, describe satellite biases in undifferenced ambiguity resolution. Second, compare CNES/CLS products to CODE products and apply both of them for Sentinel-3 satellite orbit determination. Finally, analyze Sentinel-3 satellite orbits computed by different GPS products and different data samplings.

\section{PPP-AR (ambiguity resolution)}

We describe pseudorange $(P)$ and phase $(L)$ measurements between one receiver (subscript $r$ ) and one satellite (superscript $s$ ) on frequency $l$ as

$$
\begin{aligned}
& P_{r, l}^{s}=\rho_{r, l}^{s}+c\left(d t_{\mathrm{r}}-d t^{\mathrm{s}}\right)+c\left(d_{r, l}-d_{l}^{s}\right)+I_{r, l}^{s}+e_{r, l}^{s} \\
& L_{r, l}^{s}=\rho_{r, l}^{s}+c\left(d t_{\mathrm{r}}-d t^{\mathrm{s}}\right)+c\left(b_{r, l}-b_{l}^{s}\right)-I_{r, l}^{s}+\lambda_{l} N_{r, l}^{s}+\varepsilon_{r, l}^{s}
\end{aligned}
$$

where $\rho$ represents the geometric distance, $c$ the speed of light, $d t_{\mathrm{r}}$ and $d t^{\mathrm{s}}$ the receiver and satellite clock offsets, $I$ the ionospheric delay, $\lambda$ the wavelength, $N$ the phase ambiguity, $e_{r}^{s}$ and $\varepsilon_{r}^{s}$ the other corrections and error sources of pseudorange and phase observations, for instance, tropospheric delays or multipath errors. Furthermore, $d$ and $b$ represent code and phase biases. Since $I$ is dispersive, ionosphere-free (IF) linear combination of dual-frequency measurements is widely used to eliminate the first-order ionospheric delays,

$$
\begin{aligned}
P_{r, \mathrm{IF}}^{s}= & \rho_{r, \mathrm{IF}}^{s}+c\left(\mathrm{~d} t_{r}-d t^{s}\right)+e_{r, \mathrm{IF}}^{s} \\
L_{r, \mathrm{IF}}^{s}= & \rho_{r, \mathrm{IF}}^{s}+c\left(\mathrm{~d} t_{r}-d t^{s}\right)+c\left(b_{r, \mathrm{IF}}-b_{\mathrm{IF}}^{s}\right) \\
& +\frac{1}{f_{1}^{2}-f_{2}^{2}}\left(f_{1}^{2} \lambda_{1} N_{r, 1}^{s}-f_{2}^{2} \lambda_{2} N_{r, 2}^{s}\right)+\varepsilon_{r, \mathrm{IF}}^{s}
\end{aligned}
$$


where subscript IF denotes the ionosphere-free combination. The IF code biases are assumed to be zero according to the IGS clock datum definition. Receiver phase bias $\left(b_{r, \mathrm{IF}}\right)$ is usually estimated together with receiver clock offset $\left(\mathrm{d} t_{\mathrm{r}}\right)$ as a common parameter $\left(\mathrm{d} \hat{t}_{\mathrm{r}}\right)$. In this case, the code receiver clock offset differs from that of the phase. However, the difference is within one narrow-lane cycle and can be neglected except for time transfer applications. Satellite phase bias $\left(b_{\mathrm{IF}}^{s}\right)$ is considered to be part of the ambiguity parameter in the float solution. So, in principle, there are two reasons preventing an IF ambiguity from an integer value. First, that the satellite phase bias is unknown, second, that the IF ambiguity itself cannot be expressed in the form $\lambda_{\mathrm{IF}} N_{\mathrm{IF}}$ where $N_{\text {IF }}$ is an integer ambiguity. To resolve the IF ambiguity, the widely used method is to introduce the integer wide-lane ambiguity $\left(N_{w l}=N_{1}-N_{2}\right)$ into IF ambiguity and then try to fix the deduced narrow-lane ambiguity,

$\frac{1}{f_{1}^{2}-f_{2}^{2}}\left(f_{1}^{2} \lambda_{1} N_{r, 1}^{s}-f_{2}^{2} \lambda_{2} N_{r, 2}^{s}\right)=c \frac{f_{2}}{f_{1}^{2}-f_{2}^{2}} N_{r, w l}^{s}+\frac{c}{f_{1}+f_{2}} N_{r, 1}^{s}$

where the second term $N_{r, 1}^{s}$ on the right-hand side is the narrow-lane ambiguity, with a wavelength of about $10.7 \mathrm{~cm}$ for GPS frequency $f_{1}$ and $f_{2}$. Therefore, an IF ambiguity can be resolved by fixing wide- and narrow-lane ambiguities to integer values sequentially.

To resolve wide-lane ambiguities, the Hatch-MelbourneWübbena (HMW) linear combination is widely used (Hatch 1982, Melbourne 1985; Wübbena 1985).

$$
\begin{aligned}
\operatorname{MW}\left(P_{r, 1}^{s}, P_{r, 2}^{s}, L_{r, 1}^{s}, L_{r, 2}^{s}\right) & =\frac{f_{1} L_{1}-f_{2} L_{2}}{f_{1}-f_{2}}-\frac{f_{1} P_{1}+f_{2} P_{2}}{f_{1}+f_{2}} \\
& =\lambda_{\mathrm{wl}} N_{r, \mathrm{wl}}^{s}+\lambda_{\mathrm{wl}}\left(\mathrm{mw}_{r, \mathrm{wl}}-\mathrm{mw}_{\mathrm{wl}}^{s}\right)+\varepsilon_{r, \mathrm{wl}}^{s}
\end{aligned}
$$

where $m w_{r, \mathrm{wl}}$ and $m w_{\mathrm{wl}}^{s}$ denote the HMW bias for receiver and satellite. The HMW linear combination is both geometry- and ionosphere-free. Only the ambiguity parameter and the HMW bias terms remain in the equation. To resolve the wide-lane ambiguity $N_{r, \mathrm{wl}}^{s}$, satellite HMW bias $\mathrm{mw}_{\mathrm{wl}}^{s}$ needs to be considered in advance. CNES/CLS computes daily satellite HMW biases based on GPS C1W, C2W, L1W, L2W signals. Stations with only C1C signal are corrected to C1W in advance using CODE DCB products (Dach et al. 2009). Different than that from CNES/CLS, CODE first computes OSB correction of each code signal, for instance, C1C, C1W and C2W (Villiger et al. 2019). Then, OSB corrections of $\mathrm{C} 1 \mathrm{~W}$ and $\mathrm{C} 2 \mathrm{~W}$ are applied in the HMW linear combination. The determined HMW bias products are then assumed to contain only phase biases. We need to mention that this assumption is not physically true but is consistent with satellite clock products.

While wide-lane ambiguities are fixed, narrow-lane ambiguities can be deduced by using (3). To fix narrow-lane ambiguities, we need to consider satellite phase biases $\left(b_{\mathrm{IF}}^{s}\right)$. CNES/CLS computes dedicated satellite clock products, including satellite phase biases, whereas CODE computes daily satellite narrow-lane biases $b_{I F}^{s}$ independently from satellite clock products. Then, by combining with the determined CODE satellite HMW bias products, satellite phase biases are solved on each phase signal. Details regarding CODE phase bias and integer clock estimates will be soon published by CODE (from the header of the CODE daily bias file).

\section{Comparison of CNES/CLS and CODE satellite bias products}

There are small differences when applying CODE and CNES/CLS products for PPP-AR applications. We use GPS satellite HMW biases from CNES/CLS in the HMW linear combination as

$$
\begin{aligned}
& \operatorname{MW}\left(P_{r, \mathrm{ClW}}^{s}, P_{r, \mathrm{C} 2 \mathrm{~W}}^{s}, L_{r, \mathrm{~L} 1 \mathrm{~W}}^{s}, L 2_{r, \mathrm{~L} 2 \mathrm{~W}}^{s}\right)+\lambda_{\mathrm{wl}}\left(\mathrm{mw}_{\mathrm{wl}}^{s}\right) \\
& =\lambda_{\mathrm{wl}} N_{r, \mathrm{wl}}^{s}+\lambda_{\mathrm{wl}}\left(\mathrm{mw}_{r, \mathrm{wl}}\right)+\varepsilon_{r, \mathrm{wl}}^{s}
\end{aligned}
$$

where $P_{r, \mathrm{C} 1 \mathrm{~W}}^{s}, P_{r, \mathrm{C} 2 \mathrm{~W}}^{s}, P_{r, \mathrm{~L} 1 \mathrm{~W}}^{s}, P_{r, \mathrm{~L} 2 \mathrm{~W}}^{s}$ are code and phase observations on individual signals. CNES/CLS GPS satellite HMW bias products $\left(\mathrm{mw}_{\mathrm{wl}}^{s}\right)$ are used to correct HMW measurements. The receiver HMW biases $\mathrm{mw}_{r, \mathrm{wl}}$ are estimated as epoch-wise parameters to capture the potential variation of receiver HMW biases. In this case, we need to select a reference ambiguity to cope with the singularity between receiver HMW biases and wide-lane ambiguities. The reference ambiguity is directly fixed to the closest integer value and all the other wide-lane ambiguities $\left(N_{r \text {,wl }}^{s}\right)$ can be fixed accordingly. The IF float solution using CNES/CLS products can be run in parallel to the wide-lane ambiguity resolution,

$$
\begin{aligned}
& P_{r, \mathrm{IF}}^{s}+c \mathrm{~d} \hat{t}^{s}=\rho_{r, \mathrm{IF}}^{s}+c \mathrm{~d} t_{r}+e_{r, \mathrm{IF}}^{s} \\
& L_{r, \mathrm{IF}}^{s}+c \mathrm{~d} \hat{t}^{s}-c \frac{f_{2}}{f_{1}^{2}-f_{2}^{2}} N_{r, \mathrm{wl}}^{s}=\rho_{r, \mathrm{IF}}^{s}+c \mathrm{~d} \hat{t}_{r}+\frac{c}{f_{1}+f_{2}} N_{r, 1}^{s}+\varepsilon_{r, \mathrm{IF}}^{s}
\end{aligned}
$$

where $\mathrm{d} \hat{t}^{s}$ is the CNES/CLS satellite clock product (including satellite phase bias), $\mathrm{d} \hat{t}_{r}$ includes both receiver clock offset and receiver phase bias. As we explained above (3), differences between receiver clock offset in pseudorange and phase equations are within one narrow-lane cycle and are generally tolerable considering the noise and multipath error of pseudorange measurements. Thus, we estimate a common receiver clock offset for both phase and pseudorange measurements. All the fixed wide-lane ambiguities in (5) can be introduced into the $I F$ float solution, and the deduced narrow-lane ambiguities can be resolved according to a certain 
ambiguity resolution algorithm (for instance, the LAMBDA method (Teunissen et al. 1997, 2002)).

For CODE products, we apply OSB corrections on each signal in the HMW linear combination as are discontinuous from day to day. The reason is that CNES/ CLS constrains satellite HMW biases tightly to the estimates of the previous day while CODE satellite HMW bias products seem to be independent from day to day. If we remove

$$
\begin{aligned}
& \mathrm{MW}\left(P_{r, \mathrm{C} 1 \mathrm{~W}}^{s}+\mathrm{OSB}_{\mathrm{C} 1 \mathrm{~W}}^{s}, P_{r, \mathrm{C} 2 \mathrm{~W}}^{s}+\mathrm{OSB}_{\mathrm{C} 2 \mathrm{~W}}^{s}, P_{r, \mathrm{~L} 1 \mathrm{~W}}^{s}+\mathrm{OSB}_{\mathrm{L} 1 \mathrm{~W}}^{s}, P_{r, \mathrm{~L} 2 \mathrm{~W}}^{s}+\mathrm{OSB}_{\mathrm{L} 2 \mathrm{~W}}^{s}\right) \\
& \quad=\lambda_{\mathrm{wl}} N_{r, \mathrm{wl}}^{s}+\lambda_{\mathrm{wl}}\left(\mathrm{mw}_{r, \mathrm{wl}}\right)+\varepsilon_{r, \mathrm{wl}}^{s}
\end{aligned}
$$

where OSB is the correction of satellite biases on individual signals. Then, similar as for (5) wide-lane ambiguities can be fixed to integer values. The IF float solution using CODE products is the daily datum difference (for instance using G01 as reference satellite) from CODE HMW biases and subtract integer wide-lane cycles, then the mean STD of CODE GPS satellite HMW bias products is 0.020 wide-lane cycles. Simi-

$P_{r, \mathrm{IF}}^{s}\left(P_{r, \mathrm{C} 1 \mathrm{~W}}^{s}+\mathrm{OSB}_{\mathrm{C} 1 \mathrm{~W}}^{s}, P_{r, \mathrm{C} 2 \mathrm{~W}}^{s}+\mathrm{OSB}_{\mathrm{C} 2 \mathrm{~W}}^{s}\right)+c \mathrm{~d} t^{s}=\rho_{r, I F}^{s}+c \mathrm{~d} t_{r}+e_{r, \mathrm{IF}}^{s}$

$L_{r, \mathrm{IF}}^{s}\left(L_{r, \mathrm{~L} 1 \mathrm{~W}}^{s}+\mathrm{OSB}_{\mathrm{L} 1 \mathrm{~W}}^{s}, L_{r, \mathrm{~L} 2 \mathrm{~W}}^{s}+\mathrm{OSB}_{\mathrm{L} 2 \mathrm{~W}}^{s}\right)+c \mathrm{~d} t^{s}-c \frac{f_{2}}{f_{1}^{2}-f_{2}^{2}} N_{r, \mathrm{wl}}^{s}=\rho_{r, \mathrm{IF}}^{s}+c \mathrm{~d} t_{r}+\frac{c}{f_{1}+f_{2}} N_{r, 1}^{s}+\varepsilon_{r, \mathrm{IF}}^{s}$

Satellite phase biases of each signal are corrected by OSB values. All the fixed wide-lane ambiguities in (7) are introduced as known and the deduced narrow-lane ambiguities can be fixed to integer values. We would like to mention that integer wide-lane ambiguities in (8) may differ by integer cycles compared to those in (6). Thus, this must have to be carefully considered if we intend to mix or combine the usage of CNES/CLS and CODE bias products.

The comparison of satellite HMW biases is easily possible since they are independent of satellite orbits and clock offsets. Figure 1 shows the daily GPS satellite HMW bias products from CNES/CLS (left panel), CODE (middle panel) and the difference between the two (right panel). The time interval covers doy (day of year) 266-365 2019. CNES/CLS GPS satellite HMW bias products are nearly constant over time, with a mean STD of 0.019 wide-lane cycles. In contrast, CODE GPS satellite HMW bias products larly, in the comparison of CNES/CLS and CODE HMW bias products, we remove the reference and integer-cycle differences since only the fractional part is critical for the wide-lane ambiguity resolution. As shown in the right panel, differences are very close to zero, with a mean RMS of 0.01 wide-lane cycles.

A direct comparison of satellite narrow-lane biases is not easily possible since satellite narrow-lane biases are correlated with satellite orbit and clock products, as well as with integer wide-lane ambiguities. Moreover, CNES/CLS provides no satellite narrow-lane biases but incorporates them into satellite clock offsets. We use the following method to compare CODE and CNES/CLS products in the narrow-lane ambiguity resolution. As seen from (6) and (8), different GPS satellite bias products should result in different narrowlane ambiguities.
Fig. 1 CNES/CLS, CODE (formed by OSB values) daily GPS satellite HMW biases and the fractional differences (CNES/CLS-CODE) of these two. The reference and integercycles difference are removed from CNES/CLS-CODE. For CODE products, daily integercycles and reference differences are corrected when calculating STD value of each satellite

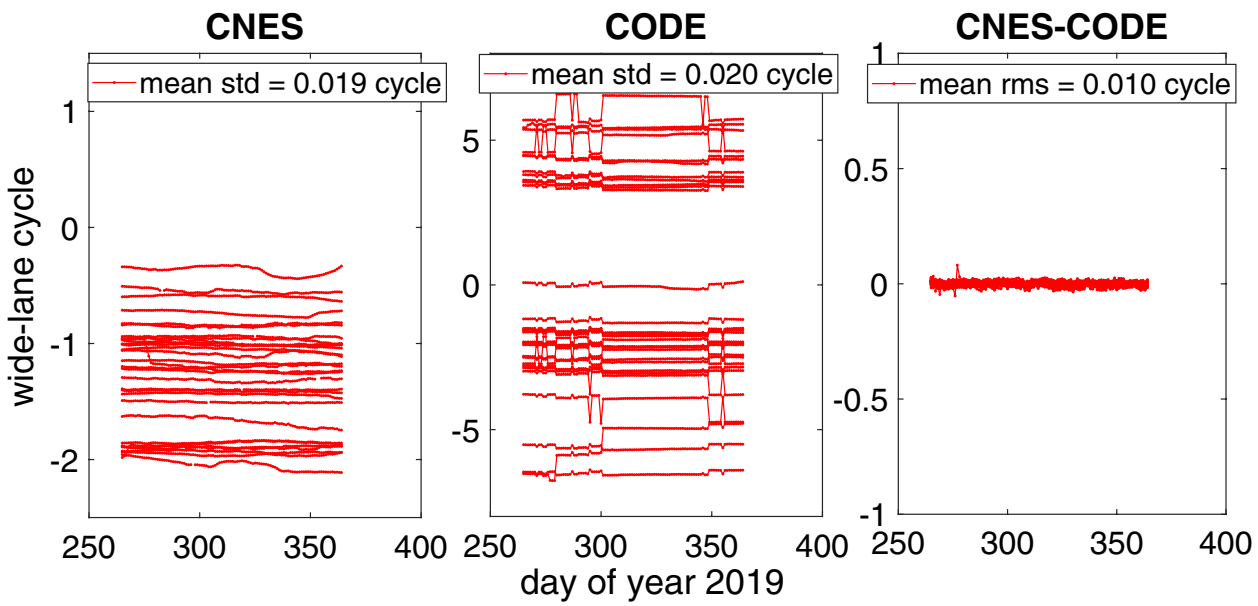




$$
\begin{aligned}
& \Delta \rho_{r, \mathrm{IF}}^{s}+c \Delta \mathrm{d} t^{s}+c \frac{f_{2}}{f_{1}^{2}-f_{2}^{2}} \Delta N_{r, \mathrm{wl}}^{s} \\
& +\mathrm{IF}\left(\mathrm{OSB}_{\mathrm{L} 1 \mathrm{~W}}^{s}, \mathrm{OSB}_{\mathrm{L} 2 \mathrm{~W}}^{s}\right) \\
& =\frac{c}{f_{1}+f_{2}} \Delta N_{r, 1}^{s}
\end{aligned}
$$

where $\Delta \rho_{r, \mathrm{IF}}^{s}$ and $\Delta \mathrm{d} t^{s}$ denote differences of satellite orbits (in the radial direction) and clock offsets, $\Delta N_{r, w l}^{s}$ denotes the difference of integer wide-lane ambiguity in cycles computed from CNES/CLS $\left(\mathrm{mw}_{\mathrm{wl}}^{s}\right)$ and CODE $\left(\mathrm{MW}\left(\mathrm{OSB}_{\mathrm{C} 1 \mathrm{~W}}^{s}, \mathrm{OSB}_{\mathrm{C} 2 \mathrm{~W}}^{s}, \mathrm{OSB}_{\mathrm{L} 1 \mathrm{~W}}^{s}, \mathrm{OSB}_{\mathrm{L} 2 \mathrm{~W}}^{s}\right)\right.$ ) bias products. $\mathrm{IF}\left(\mathrm{OSB}_{\mathrm{L} 1 \mathrm{~W}}^{s}, \mathrm{OSB}_{\mathrm{L} 2 \mathrm{~W}}^{s}\right)$ represents the IF satellite phase biases using CODE OSB products and $\Delta N_{r, 1}^{s}$ denotes the resulting narrow-lane ambiguity difference. Since satellite products from CODE and CNES/CLS have different datum, for instance satellite clock and bias products, we take $\mathrm{G} 01$ as a reference and remove the reference from all the other satellites. If the narrow-lane ambiguity of a satellite can be fixed both in (6) and (8) by using CNES/CLS and CODE products, respectively, then the sum of the left hand side of (9) should be equal to certain integer narrow-lane cycles. Therefore, the fractional part of the total difference is a representation of the difference between CNES/CLS and CODE narrow-lane phase bias. We call the fractional part of the total difference "narrow-lane" bias difference. In fact, this method can be used between two CNES/CLS-like or two CODE-like products for any other constellation as well. Comparison can be made at any sampling that is greater than or equal to that provided by the clock products. Furthermore, because the

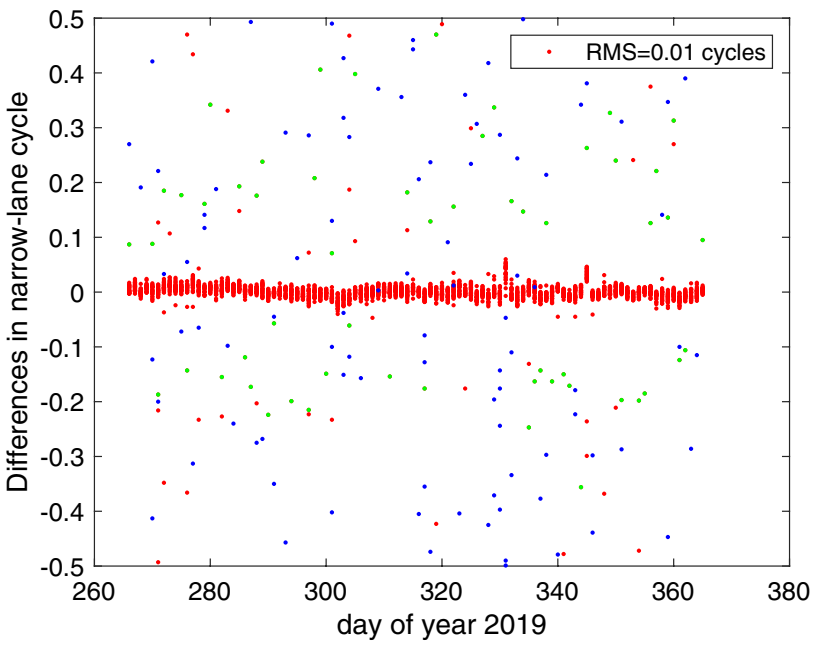

Fig. 2 GPS satellite narrow-lane bias differences between CODE and CNES/CLS products. Blue dots denote satellites for which CNES/ CLS confirmed that satellite clock products do not contain narrowlane biases. Green dots represent differences for satellite PRN 18. RMS value is the RMS of differences excluding all the outliers comparisons are solely based on satellite products, we do not need to process any measurement.

Figure 2 shows daily differences of GPS satellite narrowlane biases between CNES/CLS and CODE products. We observe some large differences every day, while most of the differences are very close to zero, with a mean RMS of 0.01 narrow-lane cycles. As informed by CNES/CLS within the CPOD QWG discussion, one or several GPS satellites in some days have no narrow-lane ambiguities fixed in the generation of satellite clock products. As a consequence, CNES/CLS satellite clock products do not contain narrowlane biases for these satellites and users should not use them for undifferenced ambiguity resolution. We display all these satellites provided by CNES/CLS with blue dots in Fig. 2, which is about $3 \%$ of the total number. Obviously, we can detect all these satellites correctly by using our comparison method, and in fact, we find more satellites with large narrow-lane bias difference. With a detailed analysis, we find that the additionally detected outliers are mainly caused by the one Block IIA satellite (PRN 18, SVN 34 with green dots in Fig. 2) as satellite clock products of this satellite cannot be well determined, especially during eclipse seasons (Duan and Hugentobler 2021). Moreover, satellite clock products of Block IIA satellites from both analysis centers are not available during post-shadow recovery periods. The amount of narrow-lane bias differences larger than 0.05 narrow-lane cycles is about $3 \%$ of the total number. $2 \%$ is caused by the Block IIA satellite, while $1 \%$ is caused by other GPS satellites. All the satellites that CNES/CLS indicates to have no narrow-lane biases are not considered in the statistics. Therefore, it is best not to use this Block IIA satellite in the ambiguity resolution.

\section{Applications in Sentinel-3 satellite POD}

As announced by Fernández (IGSMAIL-7886), precise orbit products, RINEX observation files and platform metadata of Sentinel-3 satellites were officially released on January 14, 2020. Users can access all the data from the ESA open access data Hub. As a short summary, we collect here some information that is significant for satellite POD. Sentinel-3 satellite reference frame is defined with $+X$-axis perpendicular to the launch vehicle interface plane and oriented from launch vehicle toward the satellite, $+Z$-axis parallel to the launch vehicle interface plane and pointing toward the perpendicular of the panel supporting the altimeter reflector, and Y-axis completing the right-handed orthogonal frame. Sentinel-3 satellite attitude can be determined by the 1-s sampling quaternions measured by the star trackers. The onboard GPS raw measurements are reformatted and available as RINEX file with a sampling of $1 \mathrm{~s}$. The total mass 
Table 1 Mass, center of gravity, location of GPS antenna, and phase center offset on August 9, 2019, from the Sentinel-3 POD document. $X, Y$ and $Z$ are in the satellite reference frame

\begin{tabular}{ll}
\hline Item & Value \\
\hline S3A mass & $1117.487 \mathrm{~kg}$ \\
S3b mass & $1114.430 \mathrm{~kg}$ \\
S3A center of gravity & $(X=+1.489 \mathrm{~m}, Y=+0.220 \mathrm{~m}, Z=+0.009 \mathrm{~m})$ \\
S3B center of gravity & $(X=+1.491 \mathrm{~m}, Y=+0.213 \mathrm{~m}, Z=+0.012 \mathrm{~m})$ \\
S3A GPS antenna location (GPSA receiver) & $(X=+2.881 \mathrm{~m}, Y=-0.190 \mathrm{~m}, Z=-0.794 \mathrm{~m})$ \\
S3B GPS antenna location (GPSA receiver) & $(X=+2.881 \mathrm{~m}, Y=-0.200 \mathrm{~m}, Z=-0.794 \mathrm{~m})$ \\
S3A/B GPS antenna PCV (GPSA receiver) & sen08.atx (Montenbruck et al. 2018$)$
\end{tabular}

of satellite, the center of gravity, location of GPS antenna and phase center variation (PCV) of Sentinel-3 satellites are shown in Table 1. The information on the mass varies slowly with time. Updated values can be found in the mass history file. Each Sentinel-3 satellite carries two GPS receivers and only one (GPSA) provides GPS measurements. The latest PCV values of the GPSA receiver are determined by ambiguity-fixed phase residuals (Montenbruck et al. 2018).

Optical and infrared properties of each Sentinel-3 satellite surface are given by Montenbruck et al. (2018). With this detailed information, solar radiation pressure (SRP) can be described by a box-wing macro model, as shown for one plane (Duan et al. 2020),

$\mathrm{acc}=-\frac{A}{M} \frac{S_{0}}{c} \cos \theta\left[(\alpha+\delta) e_{\mathrm{D}}+\frac{2}{3}(\delta+\kappa \alpha) e_{\mathrm{N}}+2 \rho \cos \theta e_{\mathrm{N}}\right]$

where acc represents the acceleration, $A$ the surface area, $M$ the total mass of the satellite, $S_{0}$ the solar flux, $c$ the vacuum velocity of light, $\kappa$ the thermal re-radiation factor ( 0 for solar panels and 1 for satellite body surfaces in this contribution), $\alpha, \delta, \rho$ the fractions of absorbed, diffusely scattered, and specularly reflected photons. Furthermore, $\mathbf{e}_{\mathrm{D}}$ denotes the Sun direction, $\mathbf{e}_{\mathrm{N}}$ the surface normal vector, and $\theta$ the angle between both vectors. Earth radiation is modeled similarly by using satellite infrared properties. The amount of earthreflected visible solar radiation and thermal radiation is calculated from the CERES (Clouds and Earth's Radiant Energy System) (Priestley et al. 2011). Atmospheric drag for each satellite surface is computed based on the MSISe-90 (extended Mass Spectrometer and Incoherent Scatter data) density model (Hedin 1991). Solar flux and geomagnetic activity data are obtained from the SWPC (Space Weather Prediction Center) of the US NOAA (National Oceanic and Atmospheric Administration). Orbit modeling options are given in Table 2.

We have repeated our operational processing for Sentinel3A/B satellites from doy 2662019 to doy 3652019 using CNES/CLS (grg) and CODE (cod) GPS satellite products, respectively. Satellites indicated by CNES/CLS to have no narrow-lane bias in the satellite clock products are removed from the ambiguity resolution and the associated ambiguities
Table 2 Orbit modeling options

\begin{tabular}{ll}
\hline Item & Value \\
\hline $\begin{array}{l}\text { Earth orientation } \\
\text { Attitude model }\end{array}$ & IERS 2010 (Petit and Luzum 2010) \\
Gravity field & Quaternions \\
& Measured EIGEN GL04C \\
Solid earth and ocean tides & IERS 2010 (Förste et al. 2008) \\
Ocean loading & FES2004 (Lyard et al. 2006) \\
Solar radiation pressure & Macro model \\
Earth radiation pressure & Macro model; CERES Earth radia- \\
& tion data (Priestley et al. 2011) \\
Atmospheric drag & Macro model \\
Atmospheric density model & MSISe-90 (Hedin 1991)
\end{tabular}

are estimated as float values. The Block IIA satellite PRN 18 is not used in the ambiguity resolution for both products. Settings of processing are shown in Table 3. Orbit arc length is $30 \mathrm{~h}$, with $3 \mathrm{~h}$ of the previous day and $3 \mathrm{~h}$ of the next day. Days with maneuvers and large data gaps are excluded from the analysis. One solar radiation scaling factor, one drag coefficient, and one set of sine/cose empirical parameters in alongand cross-track directions are estimated for every arc. Velocity changes are introduced every $15 \mathrm{~min}$ in radial, along- and cross-track components with a constraint of $500 \mathrm{~nm} / \mathrm{s}$. Observation sampling is $30 \mathrm{~s}$ and we set observation sampling to $10 \mathrm{~s}$ as well when using CODE products. All the processing schemes are listed in Table 4. Ambiguity fixing rates, orbit overlaps, orbit differences compared to the external combined results (COMB), and SLR residuals are taken to assess the precision and accuracy of Sentinel-3 satellite orbits.

As shown in Fig. 1, the fractional parts of HMW biases for both products are very similar. Therefore, the wide-lane fixing rates are nearly identical for cod-30 s and grg-30 s solutions, which are both more than $98 \%$. The narrow-lane fixing rates using individual products and observation samplings are shown in Fig. 3. The mean fixing rates are all above $95 \%$. There is no clear difference between grg and cod products using $30 \mathrm{~s}$ sampling observations. The cod$10 \mathrm{~s}$ results show a slightly higher narrow-lane fixing rate of about $0.5 \%$ than for $30 \mathrm{~s}$ sampling results. The reason 
Table 3 Settings of Sentinel-3 POD

\begin{tabular}{ll}
\hline Item & Value \\
\hline $\begin{array}{l}\text { Software } \\
\text { GPS products }\end{array}$ & $\begin{array}{l}\text { Bernese GNSS software 5.3 (Dach et al. 2015) modified } \\
\text { CNES/CLS orbit, HMW bias and 30-s clock; CODE satellite } \\
\text { orbit, OSB and 5-s clock products }\end{array}$ \\
GPS measurements & C1W, C2W, L1W, L2W \\
Arc length & $30 \mathrm{~h}, 3$ h of the previous day and 3 h of the next day \\
Observation sampling & $30 \mathrm{~s}$ for CODE and CNES/CLS, 10 s for CODE \\
Elevation cutoff & 0 deg \\
Solar radiation scaling factor & 1 per arc \\
Drag coefficient & 1 per arc \\
Empirical parameters & Sine/cosine terms in along- and cross-track directions per arc \\
Stochastic parameters & Velocity changes every 15 min \\
Ambiguity resolution estimator & Bernese SIGMA method (Dach et al. 2015) \\
\hline
\end{tabular}

Table 4 Processing schemes

\begin{tabular}{llll}
\hline Scheme & Sampling & GPS products & $\begin{array}{l}\text { Ambiguity } \\
\text { resolution }\end{array}$ \\
\hline grg-30 s-float & $30 \mathrm{~s}$ & CNES/CLS & No \\
grg-30 s-ambfix & $30 \mathrm{~s}$ & CNES/CLS & Yes \\
cod-30 s-float & $30 \mathrm{~s}$ & CODE & No \\
cod-30 s-ambfix & $30 \mathrm{~s}$ & CODE & Yes \\
cod-10 s-float & $10 \mathrm{~s}$ & CODE & No \\
cod-10 s-ambfix & $10 \mathrm{~s}$ & CODE & Yes \\
\hline
\end{tabular}

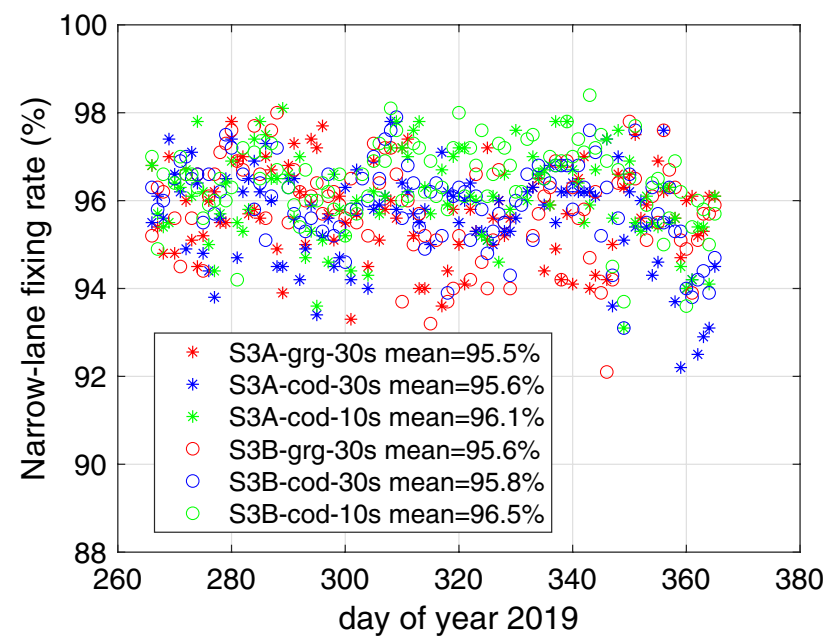

Fig. 3 Narrow-lane ambiguity fixing rates for Senitnel-3A (S3A) and Sentinel-3B (S3B) satellites using grg and cod products for the measurement sampling of $30 \mathrm{~s}$ and $10 \mathrm{~s}$

is that having more observations leads to higher reliability and robustness of parameter estimates, which is reflected by lower formal errors.

Orbit overlap comparison is traditionally employed in orbit determination to assess the internal consistency of a specific processing scheme. We compute Sentinel-3 satellite

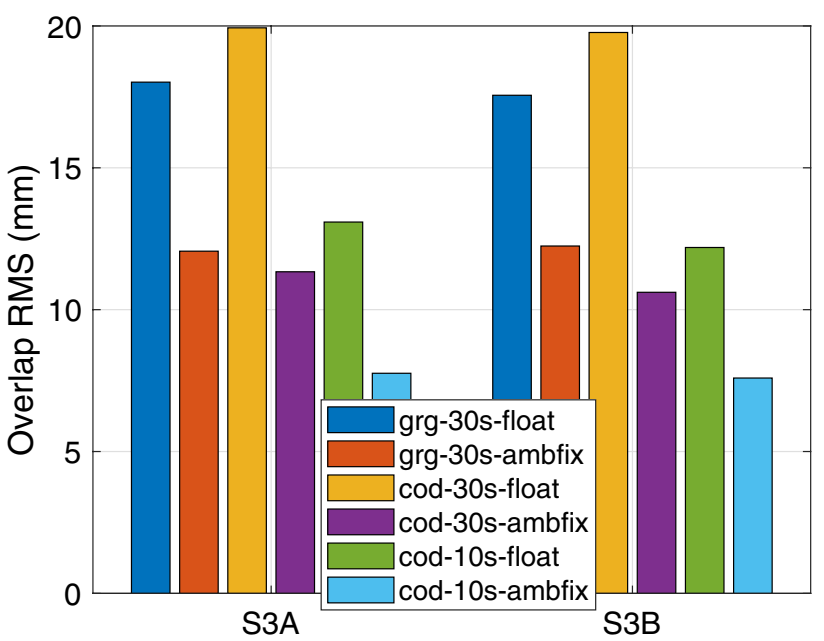

Fig. 4 6-h overlap consistency of 3D position difference for Sentinel3A/B orbit solutions (mean RMS for doy 266-365/2019)

orbits using $30 \mathrm{~h}$ of GPS measurements centered on the day of the daily solution. Each solution of one arc overlaps with the solution of the previous arc for $6 \mathrm{~h}$. The RMS of the 6-h overlap differences is computed from the 3D position differences. As shown in Fig. 4, the 3D mean RMS values for 30-s solutions using grg and cod GPS products improve from $18-20$ to $10-12 \mathrm{~mm}$ by fixing ambiguities to integer values. The cod-30 s-ambfix solution shows slightly better results than the grg-30 s-ambfix solution. This could be partly because of the usage of the same Bernese GNSS software as CODE. Both cod-10 s-float and cod-10 s-ambfix solutions show notable improvements in overlaps. However, this does not mean the corresponding orbits achieve the same improvement in precision. Because the constraints are kept fixed, the estimated pulses are relatively more determined by the observations in the case of $10 \mathrm{~s}$ sampling since 3 times more observations are then used. This results in higher variability of these pulses rendering the orbit slightly 


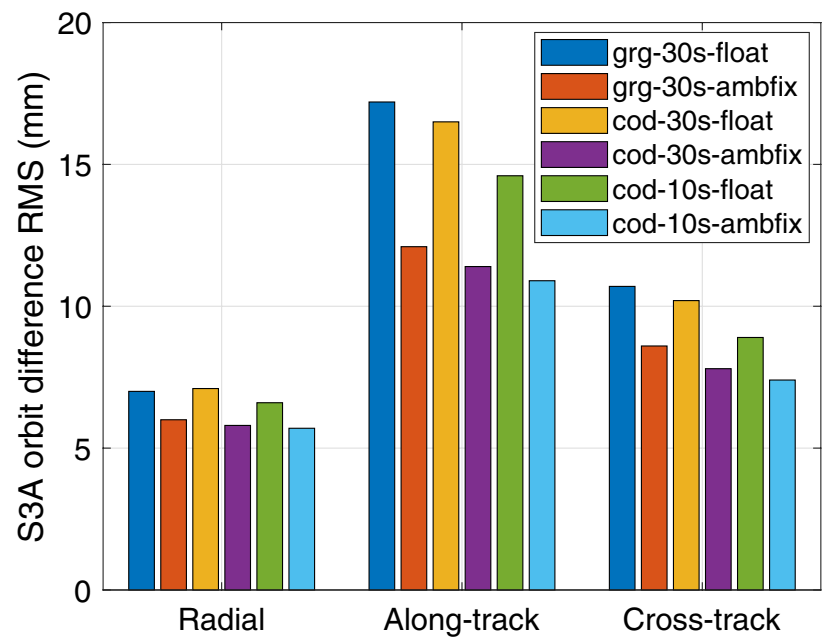

Fig. 5 Orbit differences in radial, along-track and cross-track components compared to the COMB orbits for Sentinel-3A satellite (mean RMS for doy 266-365/2019)

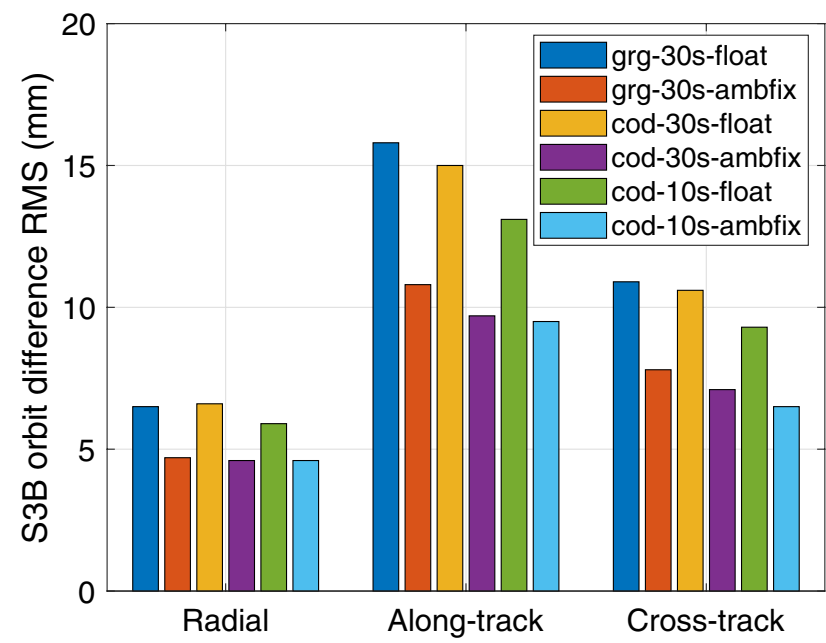

Fig. 6 Orbit differences in radial, along-track and cross-track components compared to the COMB orbits for Sentinel-3B satellite (mean RMS for doy 266-365, 2019)

less dynamic than the cod $30 \mathrm{~s}$ orbit. Therefore, a smaller overlap RMS is expected. But still, the overlap comparison between float and ambiguity-fixed solutions of the same observation sampling can be used as an indicator to assess the orbit internal consistency. RMS of orbit overlaps improves from 13 to $7 \mathrm{~mm}$ by fixing ambiguities to integer values for $10 \mathrm{~s}$ observation sampling results.

Then, we take the combined Sentinel-3 orbits (COMB) of all the Sentinel analysis centers (AIUB, CLS, CNES, CPOD, DLR, ESOC, EUM, GFZ, JPL, TUD, TUM) as external true orbits to assess the accuracy of our individual solutions. 24-h daily orbits centered on the 30-h arc are compared to the COMB orbits of the same day.
Mean RMS of orbit differences for doy 266-365/2019 is computed in radial, along- and cross-track directions, as displayed in Figs. 5 and 6. All the ambiguity-fixed solutions show notable improvements compared to the respective float solutions, especially in the along- and cross-track directions. For instance, the along-track mean RMS for the Sentinel-3A cod-30 s solution improves from 17 to $11 \mathrm{~mm}$ by fixing ambiguities to integer values. In addition, the cod$10 \mathrm{~s}$-ambfix solution shows an overall improvement of about $10 \%$ over the cod-30 s-ambfix solution. So, it is helpful to increase measurement sampling to $10 \mathrm{~s}$ if 5 -s integer GPS clock products are available.

For a fully independent assessment of the orbit quality, SLR measurements (Pearlman and Degnan 2002; Pearlman et al. 2019) are used to compare with the expected satellite-station distance based on the given orbit solutions. The nine SLR stations (except Mt. Stromlo) and the LRR (Laser Retroreflector) information are the same as given by (Montenbruck et al. 2018). The screening threshold is $10 \mathrm{~cm}$. Figures 7 and 8 show SLR residuals of Sentinel$3 \mathrm{~A}$ and Sentinel-3B satellite orbits as a function of time. Ambiguity resolution improves SLR residuals for all cases. For Sentinel-3A, the STD value is improved by about $10 \%$, $13 \%$ and $11 \%$ for grg-30 s-ambfix, cod-30 s-ambfix and cod$10 \mathrm{~s}$-ambfix solutions compared to the respective float solutions. The improvement for Sentinel-3B is about 16\%, 23\% and $13 \%$ accordingly. The cod-10 s-ambfix solution does not show clear improvement compared to the cod-30 s-ambfix solution in SLR residuals. In general, both CNES/CLS and CODE GPS products work well for ambiguity resolution in Sentinel-3 satellite orbit determination.

\section{Summary and conclusions}

In this contribution, we compare CNES/CLS satellite bias products to those from CODE and apply both of them for Sentinel-3 satellite POD. CNES/CLS provides satellite HMW biases for the wide-lane ambiguity resolution and dedicated satellite clock products (including satellite phase bias) for the narrow-lane ambiguity resolution. CODE provides satellite bias products as OSB corrections on each code and phase signal. We find that the CNES/CLS satellite HMW biases are nearly constant over time because CNES/ CLS daily HMW bias estimates are tightly constrained to those of the previous day. The CODE satellite wide-lane biases seem, however, to be independent from day to day. If we compare only the fractional part of satellite HMW biases between CNES/CLS and CODE we observe small differences, with a mean RMS of 0.01 wide-lane cycles. A direct comparison of satellite narrow-lane biases is, however, not easily possible. We compute the total difference caused by satellite orbits, clock offsets, integer wide-lane ambiguities 
Fig. 7 SLR residuals of Sentinel-3A satellite orbits for doy 266-365, 2019

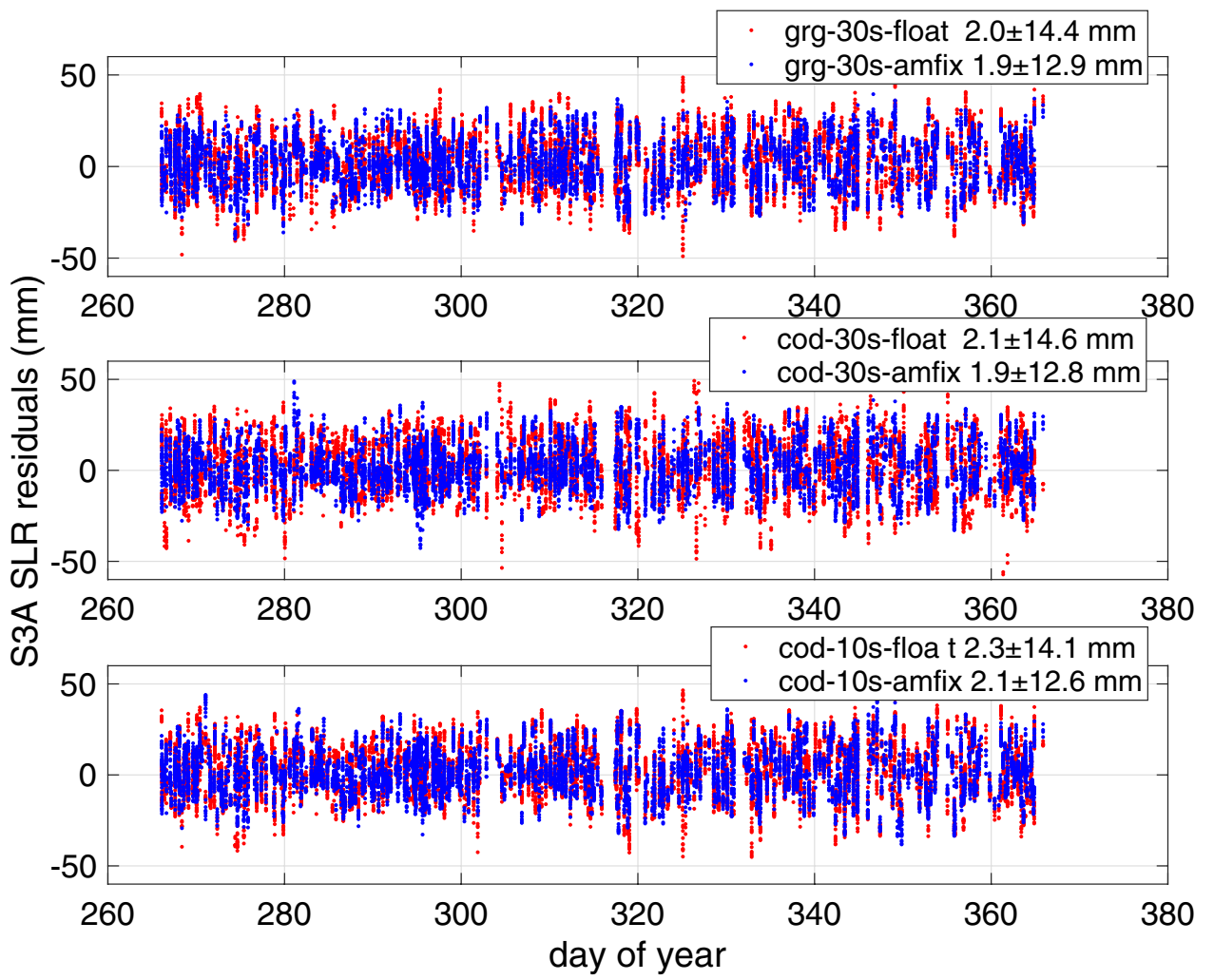

and narrow-lane biases by using different GPS satellite products in the narrow-lane equation. Then, the resulting differences of narrow-lane ambiguities represent the difference of narrow-lane biases. We find that this method can detect all those satellites that are indicated by CNES/CLS to have no narrow-lane biases in the satellite clock products. Furthermore, we find that on average $3 \%$ more satellites have differences larger than 0.05 narrow-lane cycles, with $2 \%$ caused
Fig. 8 SLR residuals of Sentinel-3B satellite orbits for doy $266-365,2019$

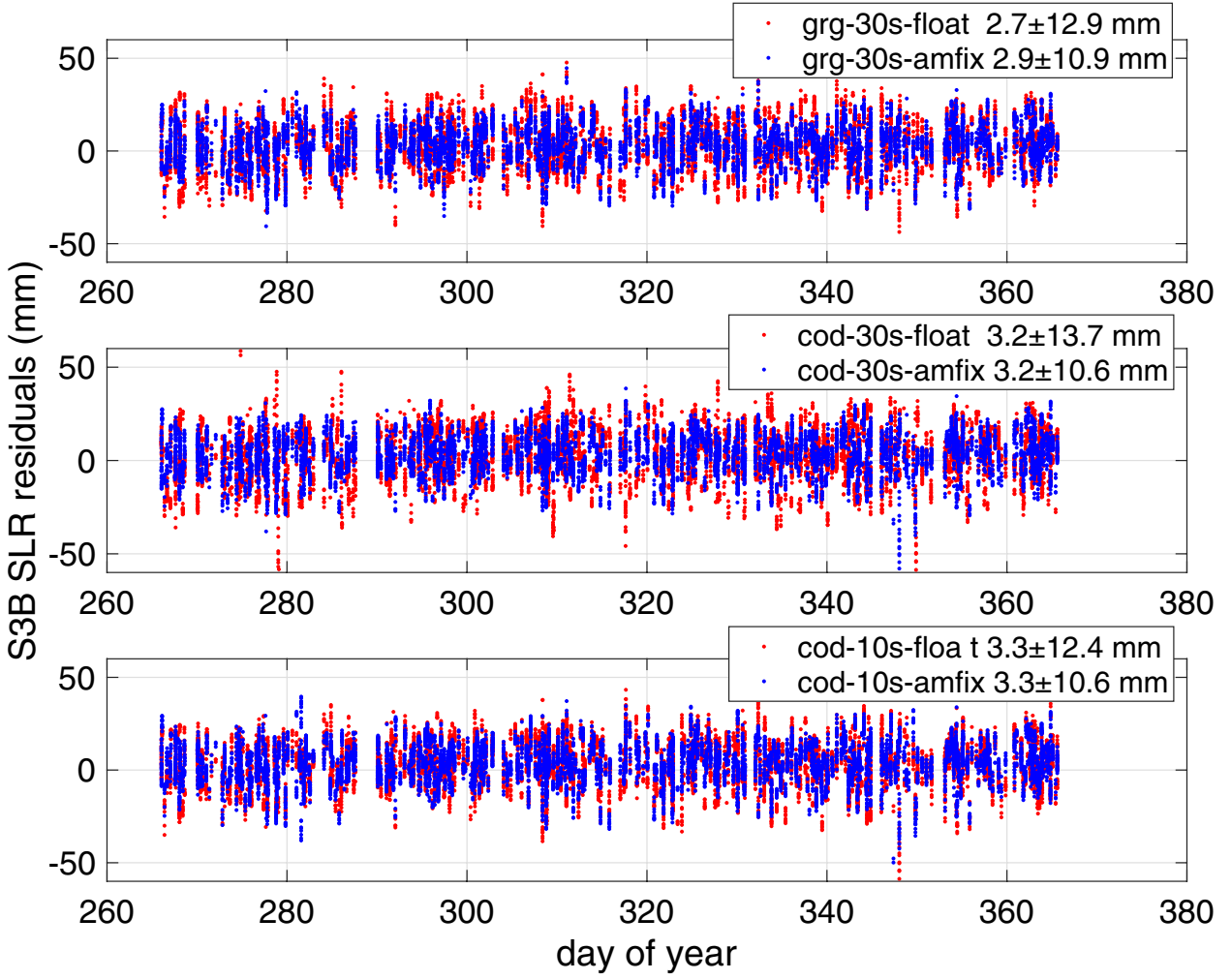


by the Block IIA satellite and $1 \%$ caused by other satellites. The reason is that Block IIA satellite clock offsets are not well determined during eclipse seasons.

Then, we apply both products for Sentinel-3 satellite POD. To avoid satellite clock interpolation, we first set observation sampling to $30 \mathrm{~s}$, the clock products sampling provided by CNES/CLS. The narrow-lane ambiguity fixing rates using CNES/CLS and CODE products are both above 95\%. 3D RMS of orbit overlap using CNES/CLS and CODE GPS products improves from 18-20 to $10-12 \mathrm{~mm}$ by fixing ambiguities to integer values. By comparing to the external COMB orbits, we observe similar improvements. When checking SLR residuals, the STD values of ambiguityfixed Sentinel-3 satellite orbits using CNES/CLS and CODE products are reduced by about $10 \%, 13 \%$ for Sentinel-3A and $16 \%, 23 \%$ for Sentinel-3B, respectively. Therefore, both CNES/CLS and CODE GPS products lead to high ambiguity success-fixing rates in Sentinel-3 satellite POD.

In addition, we process $10 \mathrm{~s}$ sampling observations using CODE products since CODE clock products have a sampling of $5 \mathrm{~s}$. The narrow-lane ambiguity fixing rate increases by $0.5 \%$ compared to that of the $30 \mathrm{~s}$ solutions. As a consequence, RMS, by comparing to the external COMB products, improves by another $10 \%$. For the SLR residuals, 10 s solutions show almost the same STD values as that of $30 \mathrm{~s}$ solutions. High sampling observations can improve orbit accuracy mainly in the along-track direction due to the slightly higher narrow-lane ambiguity fixing rate.

\begin{abstract}
Acknowledgements The Sentinel GPS observations in this study are made available by EC/ESA as part of the Copernicus program. We appreciate the continued support from GMV and CPOD QWG. GPS satellite PPP-AR products are kindly provided by the IGS CNES and CODE analysis centers. The effort of all the IGS agencies and organizations as well as all the analysis centers is acknowledged. Sentinel3A and -3B SLR observations are taken from CDDIS, we would like to thank the International Satellite Laser Ranging Service (ILRS) for making SLR measurements publicly available. Calculations are based on the Bernese GNSS Software (license available, University of Bern) and its specific modifications by the authors (not public).
\end{abstract}

Funding Open Access funding enabled and organized by Projekt DEAL.

Data availability GPS products used are publicly available from IGS CNES/CLS and CODE analysis centers. The Sentinel GPS observations in this study are made available by EC/ESA as part of the Copernicus program.

Open Access This article is licensed under a Creative Commons Attribution 4.0 International License, which permits use, sharing, adaptation, distribution and reproduction in any medium or format, as long as you give appropriate credit to the original author(s) and the source, provide a link to the Creative Commons licence, and indicate if changes were made. The images or other third party material in this article are included in the article's Creative Commons licence, unless indicated otherwise in a credit line to the material. If material is not included in the article's Creative Commons licence and your intended use is not permitted by statutory regulation or exceeds the permitted use, you will need to obtain permission directly from the copyright holder. To view a copy of this licence, visit http://creativecommons.org/licenses/by/4.0/.

\section{References}

Aschbacher J (2017) ESA's earth observation strategy and Copernicus. In: Onoda M, Young OR (eds) Satellite earth observations and their impact on society and policy. Springer, Singapore, pp 81-86. https://doi.org/10.1007/978-981-10-3713-9_5

Banville S, Geng J, Loyer S, Schaer S, Springer T, Strasser S (2020) On the interoperability of IGS products for precise point positioning with ambiguity resolution. J Geod 94(1):10. https://doi.org/10. 1007/s00190-019-01335-w

Bertiger W, Bar-Sever Y, Christensen E, Davis ES, Guinn J, Haines B, Ibanez-Meier R, Jee J, Lichten S, Melbourne W (1994) GPS precise tracking of Topex/Poseidon: results and implications. J Geophys Res: Ocean 99(C12):24449-24464

Bertiger W, Desai SD, Haines B, Harvey N, Moore AW, Owen S, Weiss JP (2010) Single receiver phase ambiguity resolution with GPS data. J Geod 84(5):327-337

Bock H, Dach R, Jäggi A, Beutler G (2009) High-rate GPS clock corrections from CODE: support of $1 \mathrm{~Hz}$ applications. J Geod 83(11):1083-1094

Bock H, Jäggi A, Beutler G, Meyer U (2014) GOCE: precise orbit determination for the entire mission. J Geod 88(11):1047-1060

Dach R, Brockmann E, Schaer S, Beutler G, Meindl M, Prange L, Bock H, Jäggi A, Ostini L (2009) GNSS processing at CODE: status report. J Geod 83(3-4):353-365

Dach R, Lutz S, Walser P, Fridez P (2015) Bernese GNSS software version 5.2, user manual. Astronomical Institute, University of Bern, Switzerland, Bern Open Publishing. https://doi.org/10. 7892/boris.72297

Duan B, Hugentobler U (2021) Enhanced solar radiation pressure model for GPS satellites considering various physical effects. GPS Solut. https://doi.org/10.1007/s10291-020-01073-z

Duan B, Hugentobler U, Hofacker M, Selmke I (2020) Improving solar radiation pressure modeling for GLONASS satellites. J Geod. https://doi.org/10.1007/s00190-020-01400-9

Duan B, Hugentobler U, Selmke I, Wang N (2021) Estimating ambiguity fixed satellite orbit, integer clock and daily bias products for GPS L1/L2, L1/L5 and Galileo E1/E5a, E1/E5b signals. J Geod. https://doi.org/10.1007/s00190-021-01500-0

Fernandez J, Ayuga F, Fernandez C, Peter H, Femenias P (2016) Copernicus POD service operations. In: Proceedings of the Sentinel-3 for Science workshop, Venice, Italy, June 2-5

Fernandez J, Escobar D, Águeda A (2014) Sentinels POD Service Operations. In: 13th international conference on space operations (SpaceOps 2014), Pasadena, California, United States, https://doi.org/10.2514/6.2014-1929

Flohrer C, Otten M, Springer T, Dow J (2011) Generating precise and homogeneous orbits for Jason-1 and Jason-2. Adv Space Res 48(1):152-172

Förste C, Schmidt R, Stubenvoll R, Flechtner F, Meyer U, König R, Neumayer H, Biancale R, Lemoine J-M, Bruinsma S (2008) The GeoForschungsZentrum Potsdam/Groupe de Recherche de Geodesie Spatiale satellite-only and combined gravity field models: EIGEN-GL04S1 and EIGEN-GL04C. J Geod 82(6):331-346

Ge M, Gendt G, Dick G, Zhang F (2005) Improving carrier-phase ambiguity resolution in global GPS network solutions. J Geod 79(1-3):103-110

Geng J, Shi C, Ge M, Dodson AH, Lou Y, Zhao Q, Liu J (2012) Improving the estimation of fractional-cycle biases for ambiguity resolution in precise point positioning. J Geod 86(8):579-589 
Hatch Ron (1982) The synergism of GPS code and carrier measurements. In: Proceedings of the third international symposium on satellite doppler positioning at physical sciences laboratory of new Mexico state university, Feb. 8-12, Vol. 2, pp 1213-1231

Hedin AE (1991) Extension of the MSIS thermosphere model into the middle and lower atmosphere. J Geophys Res Space Phys 96(A2):1159-1172

Jäggi A, Dahle C, Arnold D, Bock H, Meyer U, Beutler G, van den IJssel J, (2016) Swarm kinematic orbits and gravity fields from 18 months of GPS data. Adv Space Res 57(1):218-233

Jalabert E, Mercier F (2018) Analysis of south atlantic anomaly perturbations on Sentinel-3A ultra stable oscillator. Impact on DORIS phase measurement and DORIS station positioning. Adv Space Res 62(1):174-190

Johnston G, Riddell A, Hausler G (2017) The international GNSS service. In: Teunissen PJ, Montenbruck O (eds) Springer handbook of Global Navigation Satellite Systems. Springer, pp 967-982

Katsigianni G, Loyer S, Perosanz F, Mercier F, Zajdel R, Sośnica K (2019) Improving Galileo orbit determination using zero-difference ambiguity fixing in a Multi-GNSS processing. Adv Space Res 63(9):2952-2963

Kouba J, Héroux P (2001) Precise point positioning using IGS orbit and clock products. GPS Solut 5(2):12-28

Li X, Li X, Yuan Y, Zhang K, Zhang X, Wickert J (2018) Multi-GNSS phase delay estimation and PPP ambiguity resolution: GPS, BDS, GLONASS. Galileo J Geod 92(6):579-608

Loyer S, Perosanz F, Mercier F, Capdeville H, Marty J-C (2012) Zerodifference GPS ambiguity resolution at CNES-CLS IGS analysis center. J Geod 86(11):991-1003

Lyard F, Lefevre F, Letellier T, Francis O (2006) Modelling the global ocean tides: modern insights from FES2004. Ocean Dyn 56(5-6):394-415

Malys S, Jensen PA (1990) Geodetic point positioning with GPS carrier beat phase data from the CASA UNO experiment. Geophys Res Lett 17(5):651-654

Melbourne W The case for ranging in GPS-based geodetic systems. In: Proceedings 1st international symposium on precise positioning with the global positioning system, Rockville, Maryland, 1985. US Department of Commerce, pp 373-386

Montenbruck O, Hackel S, Jäggi A (2018) Precise orbit determination of the Sentinel-3A altimetry satellite using ambiguity-fixed GPS carrier phase observations. J Geod 92(7):711-726

Montenbruck O, Van Helleputte T, Kroes R, Gill E (2005) Reduced dynamic orbit determination using GPS code and carrier measurements. Aerosp Sci Technol 9(3):261-271

Pearlman M, Degnan JJ (2002) The international laser ranging service. Adv Space Res 30(2):135-141

Pearlman MR, Noll CE, Pavlis EC, Lemoine FG, Combrink L, Degnan JJ, Kirchner G, Schreiber U (2019) The ILRS: approaching 20 years and planning for the future. J Geod 93(11):2161-2180

Peter H, Jäggi A, Fernández J, Escobar D, Ayuga F, Arnold D, Wermuth M, Hackel S, Otten M, Simons W (2017) Sentinel-1A-First precise orbit determination results. Adv Space Res 60(5):879-892

Petit G, Luzum B (2010) IERS conventions (2010). Bureau International des Poids et mesures sevres (France). https://apps.dtic.mil/ sti/pdfs/ADA535671.pdf

Priestley KJ, Smith GL, Thomas S, Cooper D, Lee Iii RB, Walikainen D, Hess P, Szewczyk ZP, Wilson R (2011) Radiometric performance of the CERES Earth radiation budget climate record sensors on the EOS Aqua and Terra spacecraft through April 2007. J Atmos Oceanic Tech 28(1):3-21

Schaer S, Villiger A, Arnold D, Dach R, Jäggi A, Prange L (2018) New ambiguity-fixed IGS clock analysis products at CODE. In: IGS Workshop, Wuhan, China
Štěpánek P, Duan B, Filler V, Hugentobler U (2020) Inclusion of GPS clock estimates for satellites Sentinel-3A/3B in DORIS geodetic solutions. J Geod 94(12):1-20

Teunissen P, Joosten P, Tiberius C (2002) A comparison of TCAR, CIR and LAMBDA GNSS ambiguity resolution. In: Proceedings of the 15th international technical meeting of the satellite division of the institute of navigation (ION GPS 2002). pp 2799-2808

Teunissen PJ, De Jonge P, Tiberius C (1997) Performance of the LAMBDA method for fast GPS ambiguity resolution. Navigation 44(3):373-383

Uhlemann M, Gendt G, Ramatschi M, Deng Z (2015) GFZ global multi-GNSS network and data processing results. In: IAG 150 years. Springer, pp 673-679

Van IJssel DJ, Encarnação J, Doornbos E, Visser P (2015) Precise science orbits for the Swarm satellite constellation. Adv Space Res 56(6):1042-1055

Villiger A, Schaer S, Dach R, Prange L, Sušnik A, Jäggi A (2019) Determination of GNSS pseudo-absolute code biases and their long-term combination. J Geod 93(9):1487-1500

Wübbena G Software developments for geodetic positioning with GPS using TI 4100 code and carrier measurements. In: Proceedings 1st international symposium on precise positioning with the global positioning system, Rockville, Maryland, 1985. US Department of Commerce, pp 403-412

Zumberge JF, Heflin MB, Jefferson DC, Watkins MM, Webb FH (1997) Precise point positioning for the efficient and robust analysis of GPS data from large networks. J Geophy Res: Solid Earth 102(B3):5005-5017

Publisher's Note Springer Nature remains neutral with regard to jurisdictional claims in published maps and institutional affiliations.
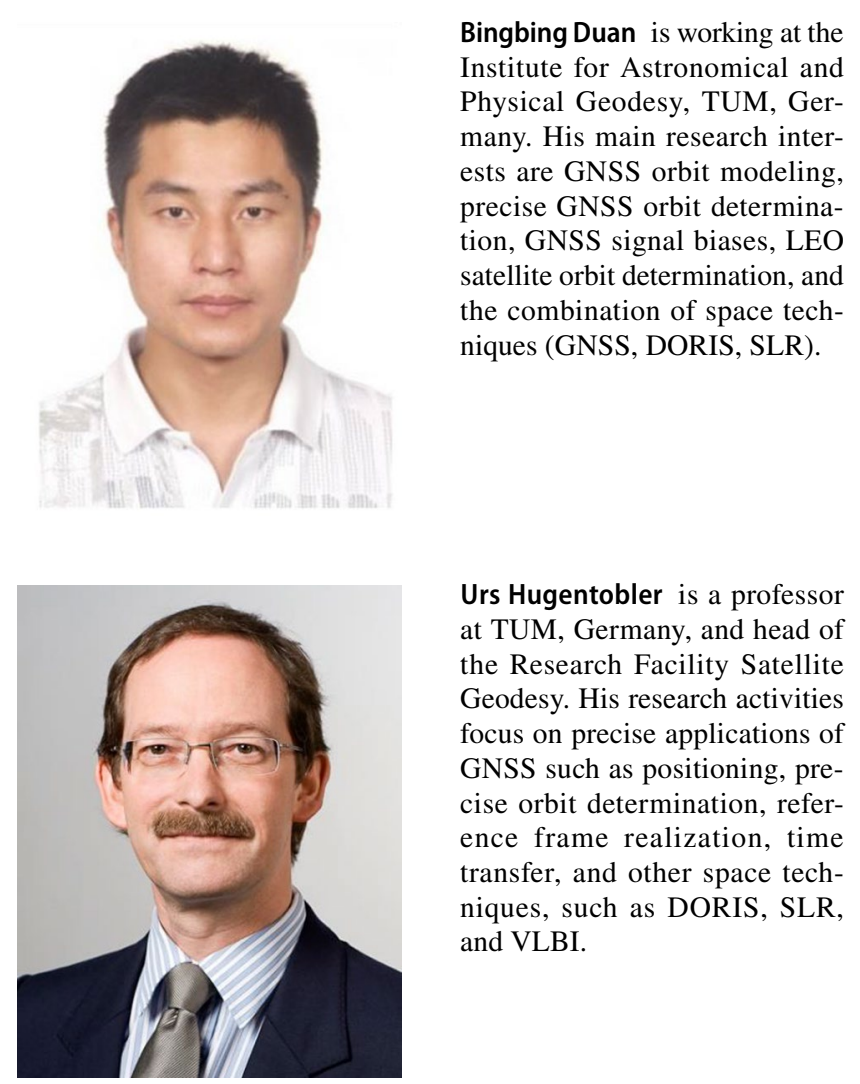

Urs Hugentobler is a professor at TUM, Germany, and head of the Research Facility Satellite Geodesy. His research activities focus on precise applications of GNSS such as positioning, precise orbit determination, reference frame realization, time transfer, and other space techniques, such as DORIS, SLR, and VLBI. 